VOL. 38 (1988) [197-202]

\title{
RECURRENCE IN LIPSCHITZ STABLE FLOWS
}

\author{
KEON-HEE LEE
}

\begin{abstract}
The purpose of this paper is to get some necessary conditions for a Poisson stable flow to be recurrent and to analyse the bilateral versions of positive and negative Lipschitz stability. Moreover, a characterisation of recurrent orbits is obtained in a certain flow.
\end{abstract}

R. Knight $[6,7]$ proved that a flow on a locally compact Hausdorff space $X$ is recurrent if and only if it is (positively, negatively) Poisson stable and each point is approximated by compact (positive, negative) weak attractors relative to its orbit closure. In this paper, to get other necessary conditions for a Poisson stable flow to be recurrent, we introduce the notions of orbitally Lipschitz stable flow, some of which are studied by Elaydi and Farran in [5]. In [5], they also showed that a flow $\pi$ on a compact manifold $M$ such that each $\pi^{t}, t \in R$, is an affine transofrmation, is positively Lipschitz stable if and only if it is negatively Lipschitz stable. However we show that the condition that $\pi^{t}$ be an affine transformation in the above results is not necessary. Finally we give some geometric properties of recurrent orbits in a certain flow.

Throughout the paper we let $(X, \pi)$ denote a flow on a locally compact metric space $X$ with a metric $d$. The orbit, orbit closure, limit set, and prolongational limit sets on $X$ are denoted, respectively, by $O, \bar{O}, L$ and $J$ with unilateral versions carrying the appropriate + or - superscript. A point $x$ in $X$ or an orbit $O(x)$ is called recurrent if and only if, given any $\varepsilon>0$, there is $T \geqslant 0$ such that $O(x) \subseteq B(y[0, T], \varepsilon)$ for any $y \in O(x)$, where $B(y[0, T], \varepsilon)=\{z \in X \mid d(y t, z)<\varepsilon$ for some $t \in[0, T]\}$. A point $\mathrm{x}$ in $X$ is said to be positively (or negatively) Poisson stable provided $x \in L^{+}(x)$ (or $x \in L^{-}(x)$ ), and $x$ is said to be (bilaterally) Poisson stable if it is both positively and negatively Poisson stable. If one of the properties above holds at each point of the phase space $X$, then flow $(X, \pi)$ is said to have that property. A set $M \subseteq X$ is called minimal if it is a closed invariant set containing no nonempty proper subset with these properties.

A flow $(X, \pi)$ is said to be (positively) Lipschitz stable at $x \in X$ if there exist $\delta(x)>0$ and $K(x) \geqslant 1$ such that $d(x t, y t) \leqslant K d(x, y)$ for all $t \in\left\{\mathbf{R}^{+}\right\} \mathbf{R}$ and all $y \in X$ with $d(x, y)<\delta$. When one can select these $\delta>0$ and $K \geqslant 1$ independently of the points $x$ in $X$, the flow $(X, \pi)$ is called (positively) Lipschitz stable. An orbit

Received 3 November 1987

Copyright Clearance Centre, Inc. Serial-fee code: $0004-9729 / 88 \quad \$ A 2.00+0.00$. 
$O(x), x \in X$, is said to be (positively) Lipschitz stable if there exist $\delta>0$ and $K \geqslant 1$ such that $d(a t, b t) \leqslant K d(a, b)$ for all $t \in\left\{\boldsymbol{P}^{+}\right\} \mathrm{P}$ and all $a, b \in O(x)$ with $d(a, b)<\delta$. Whenever each orbit of points in $X$ is (positively) Lipschitz stable, the flow $(X, \pi)$ is said to be orbitally (positively) Lipschitz stable. A flow $(X, \pi)$ is called ultimately nonexpansive provided there exists $s \in \mathbf{R}$ such that $d(x(s+t), y(s+t)) \leqslant d(x, y)$ for all $t \in \mathrm{R}^{+}$and all $x, y \in X$. The negative version of Lipschitz stability can be defined in a similiar way.

Note that $L^{+}(x)=J^{+}(x)$ if $(X, \pi)$ is (positively) Lipschitz stable at $x \in X$, but $L^{+}(x) \neq J^{+}(x)$ even if it is orbitally (positively) Lipschitz stable.

Lemma 1. Let $(X, \pi)$ be an orbitally Lipschitz stable flow. Then a point in $X$ is positively Poisson stable if and only if it is negatively Poisson stable.

PROOF: The proof is straightforward.

Theorem 2. Let $(X, \pi)$ be an orbitally (positively) Lipschitz stable flow. Then a point in $X$ is recurrent if and only if it is negatively Poisson stable.

Proof: Let $x$ be a negatively Poisson stable point in $X$. Then we shall proceed by showing that $\overline{O(x)}$ is minimal. Suppose that $y \in \overline{O(x)}$. Then it is enough to show that $\overline{O(x)} \subseteq \overline{O(y)}$, since $\overline{O(x)}$ is closed and invariant. Using the negative Poisson stability of the point $x$, we can choose a decreasing sequence $\left\{t_{n}\right\}$ in $\boldsymbol{R}^{-}$such that the sequence $\left\{x t_{n}\right\}$ converges to $y$ and $\left\{t_{n}\right\}$ approaches $-\infty$. Let $z \in \overline{O(x)}$. Then for any $\varepsilon>0$ there exists $r \in \mathbb{R}$ such that $x \in B(z, \varepsilon)(-r)$. Let us choose $\delta_{1}>0$ such that

$$
\overline{B\left(x, \delta_{1}\right)} \subseteq B(z, \varepsilon)(-r) .
$$

Since the orbit $O(x)$ is positively Lipschitz stable, there are $\delta_{2}>0$ and $K \geqslant 1$ such that

$$
d(a t, b t) \leqslant K d(a, b),
$$

for all $t \in \mathrm{R}^{+}$and all $a, b \in O(x)$ with $d(a, b)<\delta_{2}$. Put $\delta=\min \left\{\delta_{1}, \delta_{2}\right\}$. Since $\left\{x t_{n}\right\}$ converges, there exists $m$ such that $d\left(x t_{n}, x t_{m}\right)<\delta / K$ for all $n \geqslant m$. Hence we have

$$
d\left(x, x\left(t_{n}-t_{m}\right)\right) \leqslant K d\left(x t_{m}, x t_{n}\right)<\delta
$$

if $n \geqslant m$. So, $x t_{n} \in B(x, \delta) t_{m}$, for each $t_{n} \leqslant t_{m}$. Consequently we get

$$
\begin{aligned}
y \in \overline{B(x, \delta) t_{m}} & \subseteq \overline{B(x, \delta)} t_{m} \\
& \subseteq B(z, \varepsilon)(-r) t_{m}=B(z, \varepsilon)\left(t_{m}-r\right) .
\end{aligned}
$$

Thus we obtain $B(z, \varepsilon) \cap O(y) \neq \phi$. This shows that $\overline{O(x)}$ is minimal. Furthermore $\overline{O(x)}$ is compact by Theorem 12.8 in [1]. Since each orbit in a compact minimal set is recurrent, by Theorem 3.3 .8 in [2], the point $x$ is also recurrent. 
The converse is a known result for any Hausdorff phase space $X$.

We give another necessary condition for a (negatively) Poisson stable flow to be recurrent, but the proof of the theorem will be ornitted.

Theorem 3. Let $(X, \pi)$ be a (positively) lipschitz stable flow. Then a point in $X$ is recurrent if and only if it is negatively Poisson stable.

Here we give an example to show that our results make sense.

Example 4. Let $(T, \pi)$ be a flow defined on a torus by means of the planar differential system

$$
d x / d t=f(x, y), d y / d t=\alpha f(x, y)
$$

where $f(x, y)=f(x+1, y)=f(x, y+1)=f(x+1, y+1), f(x, y)>0$ if $x$ and $y$ are not both zero $(\bmod 1)$, and $f(0,0)=0$. Let $\alpha>0$ be irrational. Then the orbits of this flow $(T, \pi)$ consist of a critical point $p$ corresponding to the point $(0,0)$. Also there is exactly one orbit $O_{1}$ such that $L^{-}\left(O_{1}\right)=\{p\}$, and exactly one orbit $O_{2}$ such that $L^{+}\left(O_{2}\right)=p$. For any other orbit $O, L^{+}(O)=L^{-}(O)=T$. Furthermore $L^{+}\left(O_{1}\right)=L^{-}\left(O_{2}\right)=T$, and the flow $(T, \pi)$ does not satisfy the properties of orbitally Lipschitz stability in neighbourhoods of the critical point $p$. Moreover we have that the points on the orbit $\mathrm{O}_{2}$ are negatively Poisson stable but not positively Poisson stable, and the orbit closure $\bar{O}_{2}$ is not minimal. Consequently, we obtain that every point, except $p$, in $T$ is not recurrent, even if each point on the orbits $O$ and $\mathrm{O}_{2}$ is negatively Poisson stable.

Now we investigate the connection between positive and negative Lipschitz stability. It is clear that neither one of them implies the other even if the phase space is locally compact metric. Elaydi and Farran showed that a flow $\pi$ on a compact manifold $M$ such that each $\pi^{t}, t \in \mathrm{R}$, is an affine transformation is positively Lipschitz stable if and only if it is negatively Lipschitz stable (See [5], Theorem 2.5). However, we show that positive Lipschitz stability implies negative Lipschitz stability and vice versa, if the phase space is only compact metric.

THEOREM 5. Let $\pi$ be a flow on a compact metric space $X$. Then $(X, \pi)$ is positively Lipschitz stable if and only if it is negatively Lipschitz stable.

Proof: Assume that $(X, \pi)$ is positively Lipschitz stable, and define a map $\pi^{1}: X \longrightarrow X$ by

$$
\pi^{1}(x)=\pi(x, 1) \text { for } x \in X
$$

Then $\pi^{1}$ is a homeomorphism. Let $(X, \phi)$ be the discrete flow generated by the homeomorphism $\pi^{1}$, that is $\phi: X \times Z \longrightarrow X$ is the map defined by $\phi(x, n)=\pi^{n}(x)$ for $x \in X$ and $n \in Z$. 
First we show that the induced discrete flow $(X, \phi)$ is negatively Lipschitz stable. Since $(X, \phi)$ is positively Lipschitz stable, there exist $\delta>0$ and $K \geqslant 1$ such that if $d(x, y)<\delta$ then $d\left(\pi^{n}(x), \pi^{n}(y)\right) \leqslant K d(x, y)$ for $n=0,1,2, \ldots$ Let us introduce a new metric $\bar{d}$ on $X$ by defining

$$
\bar{d}(x, y)=\operatorname{Sup}\left\{d\left(\pi^{n}(x), \pi^{n}(y)\right): n=0,1,2, \ldots\right\}
$$

for $x, y \in X$. If $d(x, y)<\delta$ then we have

$$
d(x, y) \leqslant \bar{d}(x, y) \leqslant K d(x, y)
$$

Hence the two metrics $d$ and $\bar{d}$ on $X$ are equivalent, and so the space $(X, \bar{d})$ is also compact. Since $(X, \bar{d})$ is compact and $\bar{d}\left(\pi^{1}(x), \pi^{1}(y)\right) \leqslant \bar{d}(x, y)$, we get

$$
\bar{d}\left(\pi^{1}(x), \pi^{1}(y)\right)=\bar{d}(x, y),
$$

for $x, y \in X$. Consequently we obtain

$$
\begin{aligned}
d\left(\pi^{-n}(x), \pi^{-n}(y)\right) & \leqslant \bar{d}\left(\pi^{-n+1}(x), \pi^{-n+1}(y)\right) \\
& =\bar{d}(x, y) \\
& \leqslant K d(x, y), \text { for } n=0,1,2, \ldots,
\end{aligned}
$$

whenever $d(x, y)<\delta$. Thus $(X, \phi)$ is negatively Lipschitz stable.

Next we show that $(X, \pi)$ is also negatively Lipschitz stable. For each pair $x, y \in$ $X$, let us define

$$
S_{x y}=\operatorname{Sup}\{d(x t, y t): t \in[0,1]\}
$$

Suppose that $(X, \pi)$ is not negatively Lipschitz stable. Then we can choose $a, b \in X$ and $s \in \mathbf{R}^{-}$such that

$$
S_{a b}<\delta \text { and } d(a s, b s)>K^{2} d(a, b)
$$

where $\delta$ and $K$ are given as above. Choosing $s \in \mathbb{R}^{-}$, there exists a negative integer $m \in \mathbb{Z}^{-}$and $0 \leqslant \alpha<1$ such that $s=m+\alpha$. Let $\pi(a, \alpha)=z$ and $\pi(b, \alpha)=w$. Then we have $d(z, w) \leqslant S_{a b}<\delta$. However we get

$$
\begin{aligned}
d\left(\pi^{m}(z), \pi^{m}(w)\right) & =d(a s, b s) \\
& >K^{2} d(a, b) \\
& \geqslant K d(z, w), m \in \mathbf{Z}^{-}
\end{aligned}
$$

This is a contradiction to (1). Hence $(X, \pi)$ is negatively Lipschitz stable. 
Remark 6. Let $(X, \pi)$ be a flow, and let $(X, \phi)$ be the discrete flow generated by the homeomorphism $\pi^{1}$ as in the proof of Theorem 5 . Then it is known that $(X, \pi)$ is Liapunov stable if the induced discrete flow $(X, \phi)$ is Liapunov stable (See [3], Theorem 2.3). However we notice that $(X, \pi)$ need not be Lipschitz stable even if $(X, \phi)$ is Lipschitz stable, in particular, isometric and the phase space $X$ is compact metric.

For example, let us consider the dynamical system $\pi$ on the set $X=\{(x, y) \in$ $\left.R^{2}: x^{2}+y^{2} \leqslant 1\right\}$, generated from the differential system (polar coordinate)

$$
\left\{\begin{array}{l}
r^{3} \cos ^{2} \theta\left(r \sin \theta-\frac{r^{\prime}}{2 \pi} \cos \theta\right)=\sin \theta\left(r \cos \theta+\frac{r^{\prime}}{2 \pi} \sin \theta\right)^{2}, \\
\theta^{\prime}=2 \pi
\end{array}\right.
$$

Then the orbit $O(a, 0)$ passing through a point $(a, 0), 0<a \leqslant 1$, in $X$ is the ellipse

$$
\left\{(x, y) \in R^{2}: \frac{x^{2}}{a^{2}}+\frac{y^{2}}{a^{4}}=1\right\}
$$

and $(0,0)$ is the unique critical point of the flow. Furthermore we can see that the flow $(X, \pi)$ is not Lipschitz stable at the point $(0,0)$, even if the induced discrete flow $(X, \phi)$ is isometric.

Now we study some properties of recurrent orbits in ultimately nonexpansive flows.

Lemмa 7. Let $(X, \pi)$ be an ultimately nonexpansive flow, and let $M$ and $N$ be two nonempty disjoint compact minimal subsets of $X$. Then for any point $x$ in $M$ there exists $y$ in $N$ such that $d(M, N)=d(x, y)$.

Proof: Let $x$ be a point in a compact minimal set $M$. Then $\overline{O(x)}=M$ and $L^{+}(x)$ is a nonempty closed invariant subset of $M$. By the minimality of $M$, we have $L^{+}(x)=M$. Choose $a \in M$ and $b \in N$ such that $d(M, N)=d(a, b)$. Since $(X, \pi)$ is ultimately nonexpansive, there exists $s \in \mathbf{R}$ such that

$$
d(a(s+t), b(s+t)) \leqslant d(a, b),
$$

for all $t \in \mathbf{R}^{+}$. Since $a s \in M$ and so $x \in L^{+}(a s)$, there exists a sequence $\left\{t_{n}\right\}$ in $\mathbf{R}^{+}$ such that

$$
a\left(s+t_{n}\right) \longrightarrow x \text { as } t_{n} \longrightarrow+\infty .
$$

Since $\left\{b\left(s+t_{n}\right)\right\}$ is a sequence of points in a compact minimal set $N$, we may assume that the sequence $\left\{b\left(s+t_{n}\right)\right\}$ converges to a point, say $y$, in $N$. Consequently, for any given $\varepsilon>0$, we have

$$
\begin{aligned}
d(x, y) & \leqslant d\left(x, a\left(s+t_{n}\right)\right)+d\left(a\left(s+t_{n}\right), b\left(s+t_{n}\right)\right)+d\left(b\left(s+t_{n}\right), y\right) \\
& <\varepsilon / 2+d(a, b)+\varepsilon / 2 \\
& \leqslant d(a, b)+\varepsilon .
\end{aligned}
$$

Since $\varepsilon>0$ is arbitrary, we get $d(x, y)=d(a, b)$. 
TheOREM 8. Let $(X, \pi)$ be an ultimately nonexpansive flow, and let $p$ be a critical point in $X$. Then the closure of any other recurrent (or Poisson stable) orbit in $X$ lies on the surface of a sphere centred at $p$.

Proof: Let $O(x)$ be a recurrent (or Poisson stable) orbit in $X$ which does not contain the critical point $p$. Then we can show that the orbit closure $\overline{O(x)}$ is compact minimal as in the proof of Theorem 2. (Note that an ultimately non-expansive flow need not be (orbitally) Lipschitz stable). If we apply Lemma 7, we arrive at the results of the theorem.

\section{REFERENCES}

[1] N. Bhatia and O. Ha jek, Local semi-dynamical systems 00: Lecture Notes in Math. (Springer-Verlag, Berlin and New York, 1969).

[2] N. Bhatia and G. Szegö, Stability theory of dynamical systems (Springer-Verlag, Berlin and New York, 1970).

[3] S. Elaydi and H.R. Farran, 'On weak isometries and their embeddings in flows', Nonlinear Analysis TMA 8 (1984), 1437-1441.

[4] S. Elaydi and H.R. Farran, 'Isometries and certain dynamical systems', Bull. Austral. Math. Soc. 30 (1984), 239-246.

[5] S. Elaydi and H.R. Farran, 'Lipschitz stable dynamical systems', Nonlinear Anal. 9 (1985), 729-738.

[6] R. Knight, 'Recurrent and Poisson stable flows', Proc. Amer. Math. Soc. 83 (1981), 49-53.

[7] R. Knight, 'A characterisation of recurrent motions', Bull. Austral. Math. Soc. 28 (1983), 1-4.

Department of Mathematics

Chungnam National University

Daejeon, Korea (300-31) 